\title{
Space Weather Observations during September 2017 with CALET on the International Space Station
}

\author{
A. Bruno ${ }^{* 1}$, G. A. de Nolfo ${ }^{1}$, A. W. Ficklin ${ }^{2}$, T. G. Guzik ${ }^{2}$ and N. Cannady ${ }^{3}$ \\ ${ }^{1}$ Heliophysics Science Division, NASA Goddard Space Flight Center, Greenbelt, MD, USA \\ ${ }^{2}$ Department of Physics and Astronomy, Louisiana State University, Baton Rouge, LA, USA \\ ${ }^{3}$ Astrophysics Science Division, NASA Goddard Space Flight Center, Greenbelt, MD, USA \\ E-mail: alessandro.bruno-1enasa.gov
}

\section{for the CALET collaboration ${ }^{\dagger}$}

\begin{abstract}
A period of extreme solar activity was observed in early September 2017, during the decaying phase of solar cycle 24. A large number of bright eruptions were registered, including a X9.3 flare on 6 September and a X8.2 flare on 10 September, the two strongest soft X-ray flares in almost 11 years. Both were associated with fast Coronal Mass Ejections (CMEs) and produced Solar Energetic Particle (SEP) events measured by several spacecraft. In particular, the second event was energetic enough to induce a Ground Level Enhancement (GLE) detected by the worldwide neutron monitor network, the second GLE of solar cycle 24. In this work we present a preliminary analysis of the September 2017 SEP events made with the CALorimetric Electron Telescope (CALET) onboard the International Space Station (ISS). We also investigate the relativistic electron precipitation (REP) events associated with the geomagnetic storms occurring in the same period. Data are compared with those of other space- and ground-based detectors.
\end{abstract}

36th International cosmic-ray Conference -ICRC2019-

July 24th - August 1st, 2019

Madison, WI, U.S.A.

\footnotetext{
* Speaker.

${ }^{\dagger}$ for collaboration list see PoS(ICRC2019)1177
} 


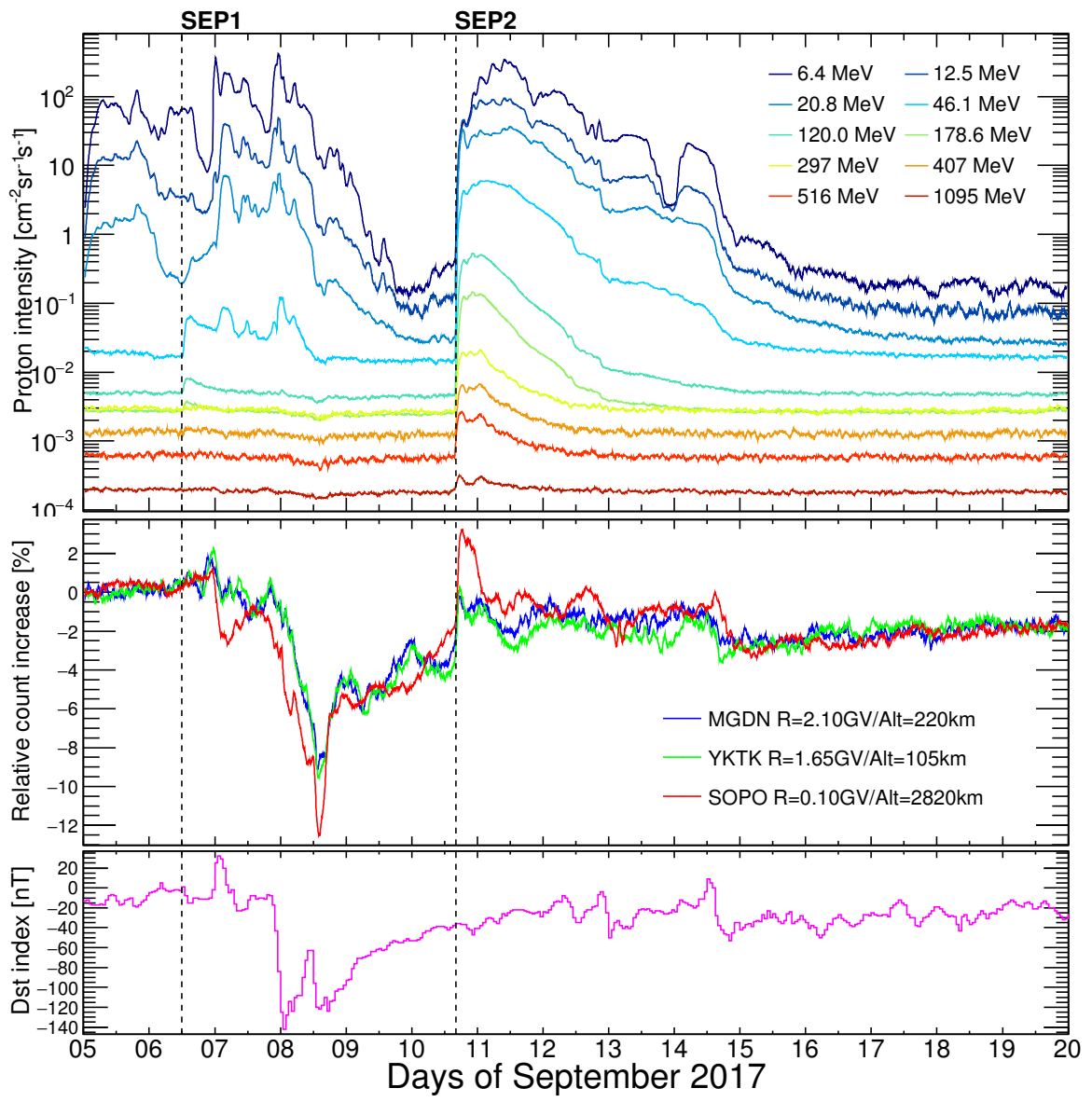

Figure 1: Time profiles (5-19 September 2017) of proton intensities measured by GOES-15 (top panel, 1-hour running averages), relative count-rate increases registered by the SOPO, YKTK and MGDN neutron monitor stations at different geomagnetic cutoff rigidities / altitudes (middle panel, 1-hour running averages), and $D s t$ index (bottom panel, 1-hour resolution). The vertical dashed lines mark the onset of the 6 and 10 September SEP events.

\section{Introduction}

An exceptional solar activity was registered in the first half of September 2017, with a large number of powerful eruptions originating from the Active Region 12673. In particular, the two flares on 6 September (X9.3) and 10 September (X8.2) were the brightest soft X-ray flares observed since December 2006. Interestingly, both extreme solar activity periods occurred late in the decay phase of respective solar cycles. The 6 and 10 September eruptions, associated with fast halo coronal mass ejections (CMEs) with $1571 \mathrm{~km} / \mathrm{s}$ and $3163 \mathrm{~km} / \mathrm{s}$ sky-plane speeds (https: //cdaw.gsfc.nasa.gov/CME_list/), respectively, were magnetically connected to Earth, inducing solar energetic particle (SEP) events detected by several spacecraft [1].

The temporal variations of proton intensities measured by the EPEAD/HEPAD instruments on board GOES-15 between 5-19 September are displayed in the top panel of Figure 1; the energy values reported for each proton channel are based on the calibrations by [2, 3]. The dashed vertical lines mark the onset of the two SEP events on 6 and 10 September. The former 

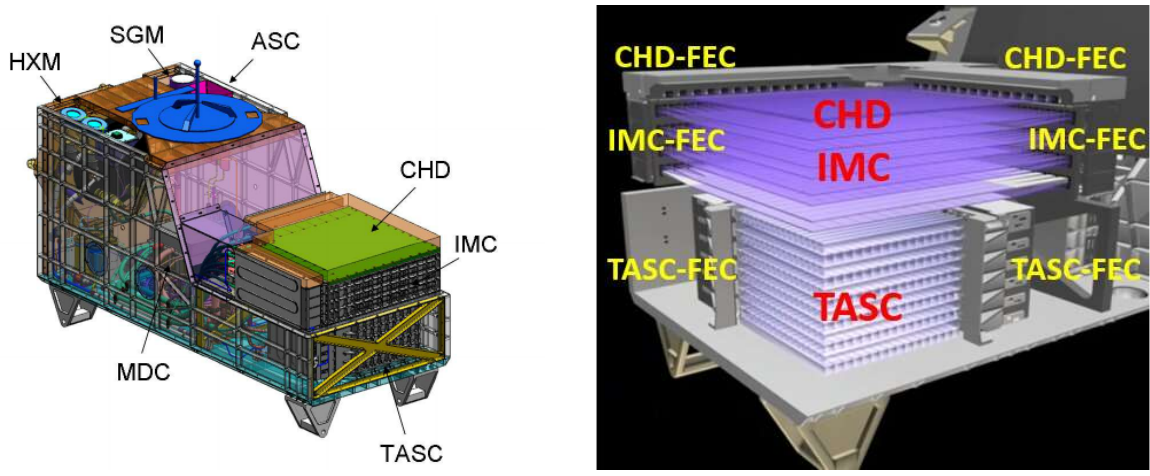

Figure 2: Left: schematic view of the CALET instrument showing the main calorimeter, the Gamma-ray Burst Monitor (CGBM) consisting of a hard X-ray monitor, and a soft gamma-ray monitor, Mission Data Controller (MDC) and support sensors, including Global Position Sensor Receiver (GPSR) and Advanced Stellar Compass (ASC) as indicated in the figure. Right: layout of the main calorimeter, which consists of a Charge Detector (CHD), an IMaging Clorimeter (IMC), and Total AbSorption Calorimeter (TASC), where FEC stands for front end electronics.

was limited to energies below a few hundred $\mathrm{MeV}$. The latter was energetic enough to trigger a Ground Level Enhancement ${ }^{1}$ (GLE), the second of solar cycle 24, detected by several neutron monitors (NMs). The middle panel of Figure 1 shows the relative count-rates of the South Pole (SOPO), Yakutsk (YKTK) and Magadan (MGDN) stations at different detection thresholds ${ }^{2}$ (http://www.nmdb.eu/nest/). It was a relatively small GLE, as the maximum increase in the SOPO count-rates was $\sim 6 \%$. It commenced in the recovery phase of a Forbush decrease induced by the arrival of a complex interplanetary shock-ICME ${ }^{3}$ structure late on 7 September, followed by another ICME passing Earth about 12 hours later. These solar wind disturbances, associated with previous eruptions on 6 September, also caused a major multiple geomagnetic storm, as indicated by the large variations (down to $-142 \mathrm{nT}$ ) of the Disturbance Storm Time (Dst) index reported in the bottom panel of Figure 1. In general, the near-Earth environment in early September 2017 was significantly affected by a number of interplanetary transients (shocks, ICMEs and highspeed streams (HSSs)), possibly influencing the magnetic connectivity to the Sun and resulting in an enhanced magnetospheric activity [1]. In particular, a moderate storm commenced late on 12 September after the passage of the eastern flank of the shock associated with the 10 September event, and a long-duration storm interval was driven by a HSS arriving on 14 September.

\section{The CALET experiment}

The CALorimetric Electron Telescope (CALET) [4], installed on the International Space Station (ISS) in August 2015, has been accumulating scientific data since October 2015. CALET is primarily intended to investigate nearby cosmic-ray accelerators and search for dark matter by

\footnotetext{
${ }^{1}$ According to convention, a SEP event is counted as a GLE if at least two independent NMs - including a near sea level station - have registered a simultaneous statistically significant increase related to the SEP arrival.

${ }^{2}$ We note that, for the high altitude SOPO station, the effective detection threshold is actually controlled by the atmospheric absorption ( $\sim 300 \mathrm{MeV})$ rather than geomagnetic effects $(\mathrm{R} \sim 0.1 \mathrm{GV})$.

${ }^{3}$ The interplanetary counterparts of CMEs are denoted as "ICMEs".
} 

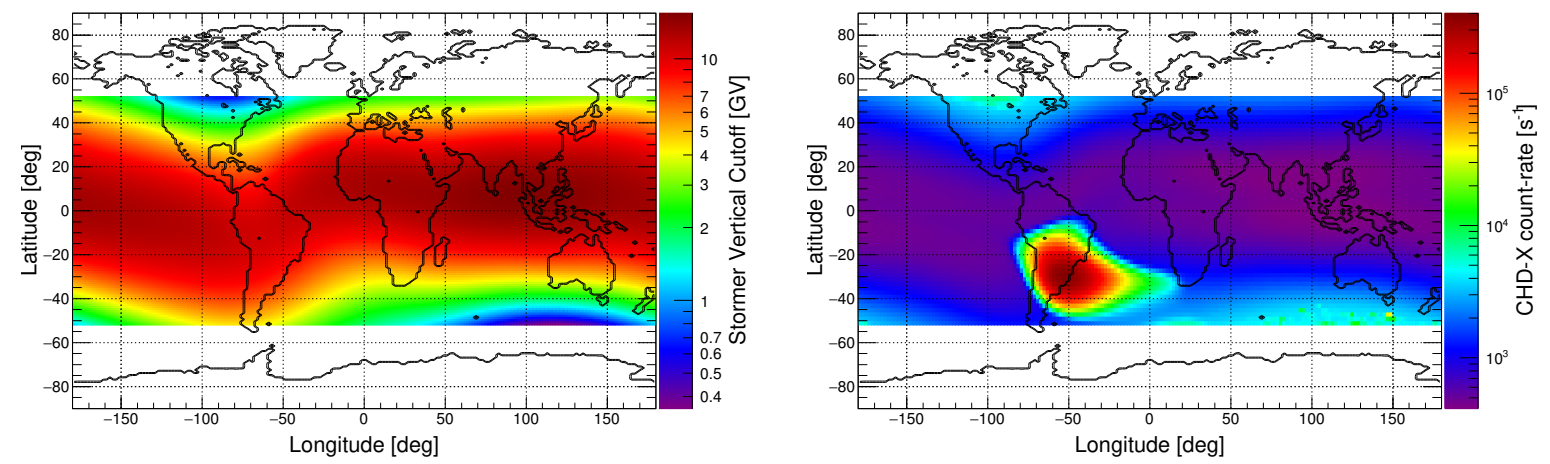

Figure 3: Left: Störmer vertical cutoff rigidity (color code) as a function of geographic coordinates for the ISS orbit during a period of quiet solar activity in August 2017. Geomagnetic data are based on the IGRF-12 and TS05 magnetic models. Right: count-rate (color code) as a function of geographic coordinates measured by the CALET top detector (CHD-X) during a period of quiet solar activity in August 2017.

precisely measuring all-electron $\left(\mathrm{e}^{-}\right.$and $\left.\mathrm{e}^{+}\right)$and gamma-ray spectra in a wide energy range from $1 \mathrm{GeV}$ to $20 \mathrm{TeV}$. In addition, CALET can measure the energy spectra of protons, heliums, and heavier nuclei up to $\sim 1 \mathrm{PeV} / \mathrm{n}$, and the ultra-heavy $(\mathrm{Z}>28$ ) nuclei composition above $600 \mathrm{MeV} / \mathrm{n}$.

A schematic overview of the instrument is shown in the left panel of Figure 2, with the main components given by the electromagnetic calorimeter, displayed in the right panel, and the gammaray burst monitor. The CALET calorimeter is a large-area high-performance instrument composed of a charge detector (CHD), a pre-shower imaging calorimeter (IMC) and a total absorption calorimeter (TASC). The CHD is a silicon detector array providing the necessary charge resolution for the measurement of light and heavy nuclei. The IMC is a finely segmented sampling calorimeter used to determine the incoming particle trajectory, supporting the measurement of the particle shower by the TASC. The latter is a homogeneous calorimeter with a 27 radiation-length thickness, which completely absorbs the electron shower energy in the TeV energy range and separate electrons from the overwhelming flux of protons. Details about the apparatus and its performance can be found elsewhere (e.g. [4, 5] and references therein). By combining its unique identification capabilities and the long-term observations enabled by the installation on the ISS, CALET is expected to extend the limits of previous space-based experiments.

In addition to the aforementioned astrophysics goals, CALET is able to provide a continuous monitoring of space weather phenomena affecting the near-Earth environment, including SEP events and relativistic electron precipitation (REP) events [6].

\section{CALET observations}

The cosmic-ray access to the Earth's magnetosphere is commonly quantified in terms of the Störmer cutoff rigidity [7], whose analytical expression for vertically incident particles is given by $R=K / L^{2}[\mathrm{GV}]$, where $L$ is the Mcllwain's parameter ( $L$-shell, measured in Earth radii $\left[\mathrm{R}_{E}\right]$ ) and the constant $K$ is related to the terrestrial magnetic dipole moment ${ }^{4}\left(K \sim 14.2 \mathrm{R}_{E}^{2} \mathrm{GV}\right.$ in 2017). The geomagnetic cutoff map estimated for the ISS orbit - averaged over altitudes - during a period of

\footnotetext{
${ }^{4}$ Note that $K$ is time-dependent due to geomagnetic secular variations.
} 
relatively quiet solar activity (August 2017) is reported in the left panel of Figure 3; the calculation, based on the International Geomagnetic Reference Field-12 (IGRF-12) [8] and the Tsyganenko \& Sitnov (TS05) [9] models, accounts for the effects of both internal and external geomagnetic field sources. Since the ISS orbital inclination is $\sim 51.6 \mathrm{deg}$, the average $R$ value at highest geographic latitudes is relatively high $(\sim 2.3 \mathrm{GV})$; the minimum $R(\sim 0.33 \mathrm{GV})$ is occasionally reached when the spacecraft is closest to the North magnetic pole in the southern geographic hemisphere ${ }^{5}$. As a consequence, CALET is sensitive to the relatively high-energy SEPs, taking advantage of its large geometric factor. On the other hand, the minimum energy for REP events is determined by the detector discriminator threshold ( $\sim 1.6 \mathrm{MeV}$ for CHD-X).

The average count-rate of the CALET top silicon plane (CHD-X) during August 2017 is displayed in the right panel of Figure 3. It peaks in the region of the so-called South Atlantic Anomaly (SAA), where the inner Van Allen belt (mostly populated by geomagnetically trapped protons) makes its closest approach to the Earth's surface ${ }^{6}$. Minor count-rate enhancements are registered at highest magnetic latitudes due to low-energy interplanetary particles and outer belt electrons.

Figure 4 reports the CALET space weather observations during early September 2017, as a function of $L$ and the local geomagnetic field strength (B), for four different time intervals (see labels). The high-rate area at $B \lesssim 0.24 \mathrm{G}$ and $L \lesssim 2.3 \mathrm{R}_{E}$ corresponds to the SAA. The top-left panel shows the count-rate registered by CHD-X between 1-6 September, prior to the SEP events. The top-right panel is relative to the first SEP event occurring on 6 September at $~ 12 \mathrm{UT}$ : a small increase can be observed at high $L$-shells (high magnetic latitudes). The enhancement at $L \sim 3-5 \mathrm{R}_{E}$ (outer belt boundaries) registered between 7-10 September (bottom-left panel) appears to be related to an electron precipitation (REP event) associated with the major geomagnetic storm caused by the interplanetary disturbances passing Earth between the two SEP events. Finally, the bottomright panel displays the count-rate distribution during the 10 September SEP event, commencing at 16UT: a significant increase due to solar protons is measured at high magnetic latitudes; a REP component in the outer belt region is still present (geomagnetic storms after 12 and 14 September).

Figure 5 shows the 10-min resolution profiles of count-rates measured between 6-19 September by three CALET detector layers: CHD-X, IMC-X1 and IMC-Y4 (i.e. the first and last IMC planes), characterized by different penetration energies. Data acquired during the passage of the ISS in the SAA were discarded. Each color corresponds to a bin of $L$-shell or, equivalently, to a bin of $R$, so that the geomagnetic cutoff information can be used to infer the minimum rigidity of SEPs. A data gap is present between 4-5 September.

The 6 September SEP event resulted in a small count-rate enhancement, in agreement with GOES observations reporting a statistically significant signal limited to energies below $\sim 200 \mathrm{MeV}$ [1]. In contrast, the 10 September SEP event, characterized by protons with energies exceeding the sea-level NM detection threshold $(\sim 500 \mathrm{MeV})$, produced a much higher count-rate increase which lasted for several days. However, since at the onset time ( $\sim 16$ UT) the ISS was located at relatively high geomagnetic cutoff regions, CALET did not observe the arrival of $\lesssim 2 \mathrm{GeV}$ solar protons during the initial phase of the event; in particular, $R$ fell below $1 \mathrm{GV}$ after $\sim 03: 30 \mathrm{UT}$ on

\footnotetext{
${ }^{5}$ Note that magnetic and geographic North poles are located in opposite hemispheres.

${ }^{6}$ The SAA is a consequence of the tilt $(\sim 11 \mathrm{deg})$ between the magnetic dipole axis of the Earth and its rotational axis, and of the offset $(\sim 500 \mathrm{~km})$ between the dipole and the Earth centers.
} 

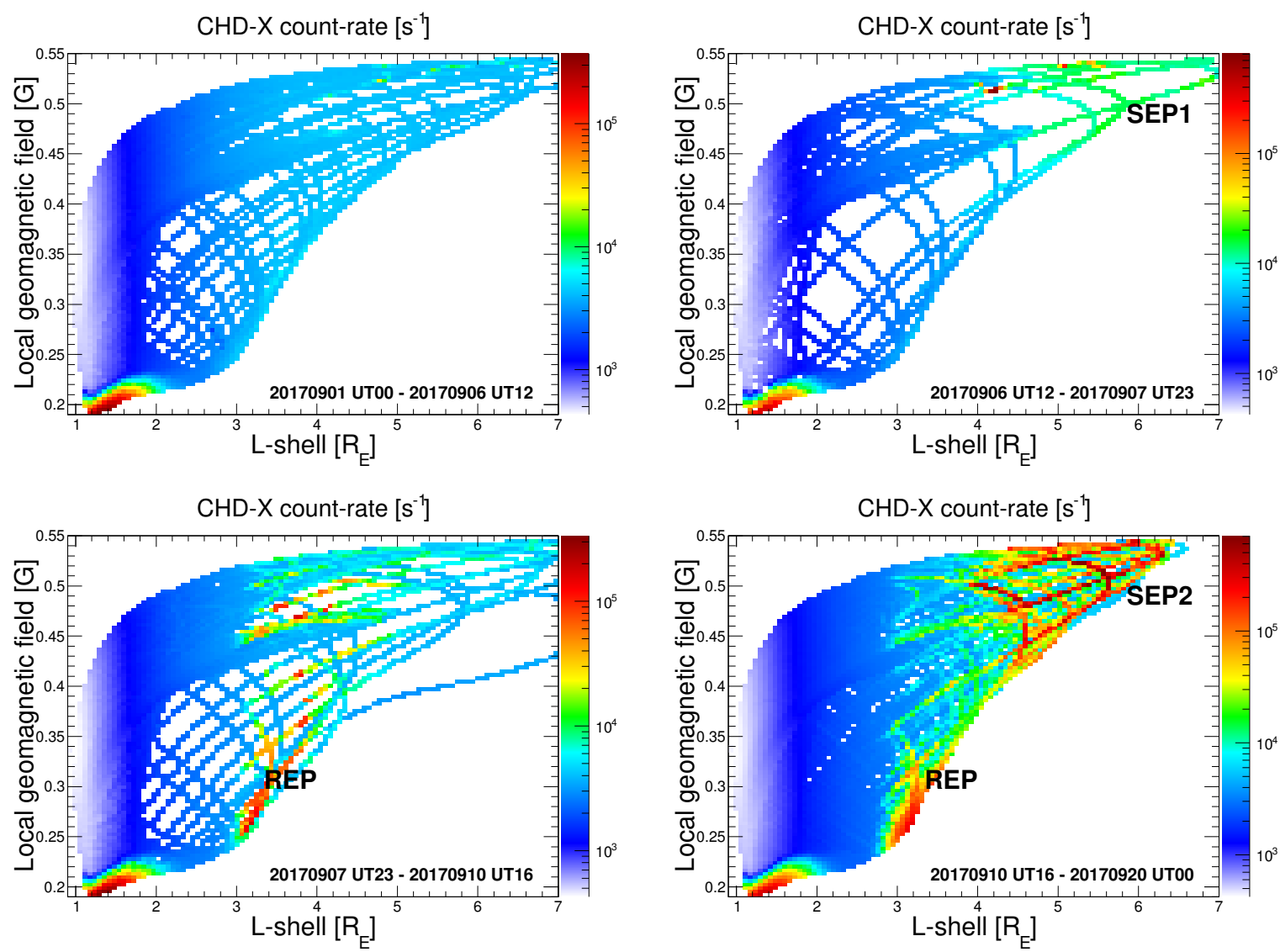

Figure 4: Count-rate (color code) measured by the CALET top detector (CHD-X) as a function of local geomagnetic field intensity $B$ and McIlwain's $L$-shell, during four consecutive time intervals in early September 2017 (see labels). The enhancements relative to SEP and REP events are indicated.

11 September. Furthermore, the statistical significance of the SEP signal above several hundred $\mathrm{MeV}$ is quite small, consistent with the relatively soft spectrum measured in this range [1].

Both SEP events were followed by REP events registered at $L$-shell values between $\sim 3-5$ $\mathrm{R}_{E}$, mostly in association with the geomagnetic storms occurring after 7, 12 and 14 September, as shown in bottom panels of Figure 4. For a comparison, Figure 6 displays the time profile of the 2 $\mathrm{MeV}$ electron intensity as a function of $L$ measured by the Relativistic Electron Proton Telescope (REPT) onboard the Van Allen Probes (http://rbspgway. jhuapl.edu/), along with the Dst index variations. The REPT data show a clear enhancement of the outer radiation belt at the same $L$-shells related to the significant geomagnetic activity registered in those days, in particular during the recovery phases of the two major storms commencing on 7 and 14 September. A part of the trapped electrons reached down to ISS altitudes probably due to enhanced plasma wave activities in the inner magnetosphere, causing the REP events.

\section{Conclusions}

The CALET instrument onboard the ISS is able to provide a continuous monitoring of space 


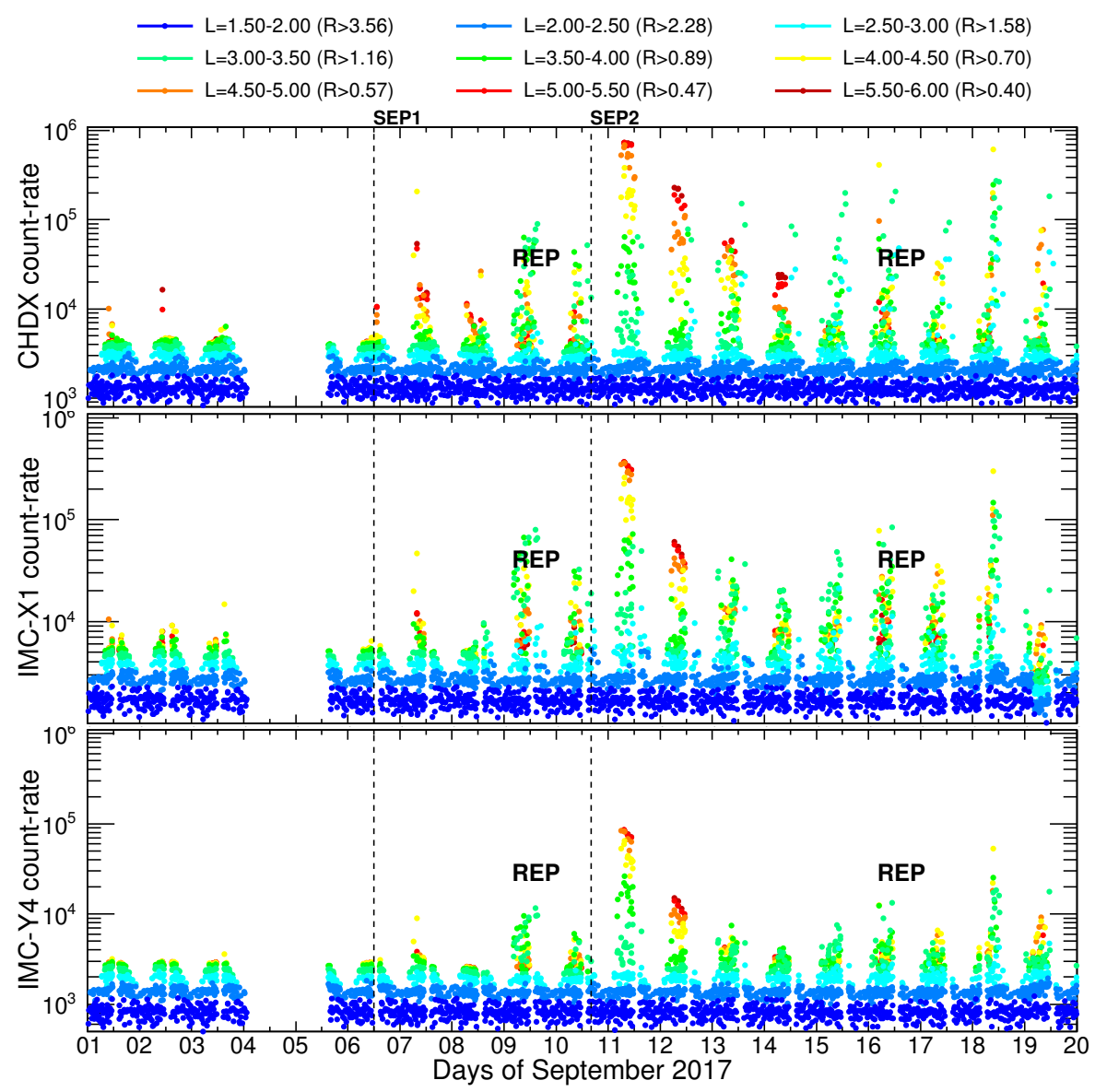

Figure 5: Count-rates of selected CALET detector planes (CHD-X: top, IMC-X1: middle, IMC-Y4: bottom) between 1-19 September 2017, for different bins of McIlwain's $L$-shell $\left(\mathrm{R}_{E}\right.$, color code); the corresponding Stormer vertical cutoff rigidity (GV) is reported as well. The two vertical dashed lines denote the onset of two SEP events on 6 and 10 September. The REP events $\left(L \sim 3-5 \mathrm{R}_{E}\right)$ are also indicated.

weather phenomena affecting the near-Earth environment, including SEP and REP events. Due to the relatively high geomagnetic cutoff associated with its orbit, CALET's sensitivity to SEPs is limited to most energetic events, taking advantage of the large geometric factor. In this work we presented a preliminary measurement of the SEP events occurring in early September 2017, late in the decay phase of the solar cycle 24. The period was characterized by an extreme solar activity, with a number of geo-effective eruptions. CALET observations include both the small SEP event on 6 September, limited to energies below few hundreds of $\mathrm{MeV}$, and the much more energetic event on 10 September, which resulted in a GLE. In addition, we reported the detection of multiple REP events occurring in the same interval in association with the significant geomagnetic activity induced by the arrival of several solar wind disturbances.

\section{Acknowledgements}

A. B. acknowledges support by an appointment to the NASA postdoctoral program at the NASA Goddard Space Flight Center administered by Universities Space Research Association under contract with NASA. 


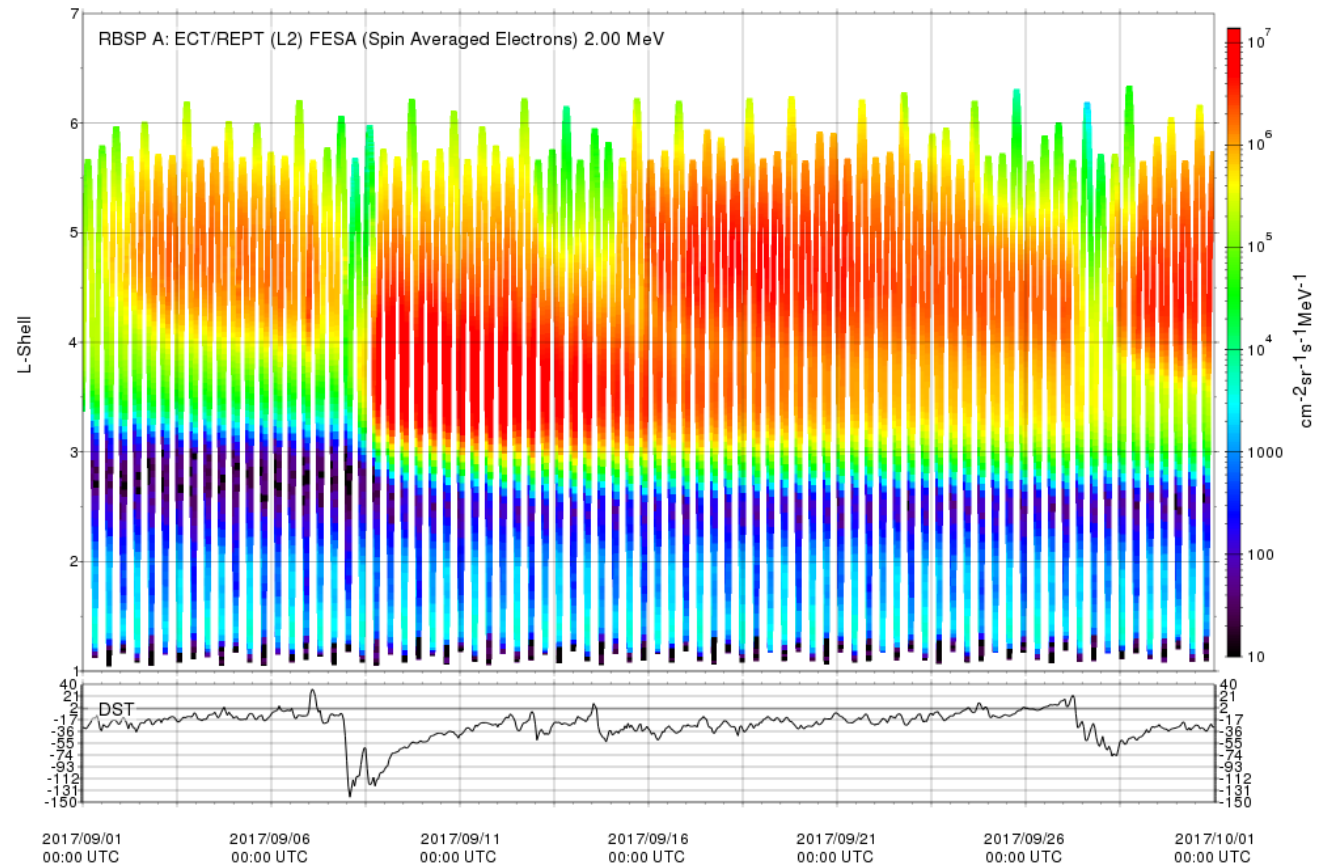

Figure 6: Top: time variations of $2 \mathrm{MeV}$ electron intensities as a function of $L$-shell measured by the REPT instrument onboard the Van Allen Probe A (RBSP A) during September 2017 (data from http://rbspgway.jhuapl.edu/). Bottom: temporal profile of the Dst index during the same period.

\section{References}

[1] A. Bruno, et al. 2019, Spectral Analysis of the 2017 September Solar Energetic Particle Events, Space Weather, 17, 419, https://doi.org/doi:10.1029/2018sw002085

[2] I. Sandberg, et al. 2014, Cross calibration of NOAA GOES solar proton detectors using corrected NASA IMP-8/GME data, Geophys. Res. Lett., 41, 4435, https : / / doi .org/10 .1002/2014 GL060469

[3] A. Bruno 2017, Calibration of the GOES 13/15 high-energy proton detectors based on the PAMELA solar energetic particle observations, Space Weather, 15,1191, https://doi.org/10.1002/2017SW001672

[4] S. Torii, et al. (CALET Collaboration) 2016, The calorimetric electron telescope (CALET): a high-energy astroparticle physics observatory on the international space station, Proceeding of Science (ICRC2015), 236, 581, https://doi.org/10.22323/1.236.0581

[5] O. Adriani, et al. (CALET Collaboration) 2019, Direct Measurement of the Cosmic-Ray Proton Spectrum from $50 \mathrm{GeV}$ to $10 \mathrm{TeV}$ with the Calorimetric Electron Telescope on the International Space Station, Phys. Rev. Lett. 122,181102, https://doi.org/10.1103/PhysRevLett.122.181102

[6] R. Kataoka, et al. 2016, Relativistic electron precipitation at International Space Station: Space weather monitoring by Calorimetric Electron Telescope, Geophys. Res. Lett., 43, 4119, https://doi.org/10.1002/2016GL068930

[7] C. Störmer 1955, The Polar Aurora, Oxford Univ. Press, London, England

[8] E. Thébault, et al. 2015, International Geomagnetic Reference Field: the 12th generation, Planets and Space, 67:79, https://doi.org/10.1186/s40623-015-0228-9

[9] N. A. Tsyganenko \& M. I. Sitnov 2005, Modeling the dynamics of the inner magnetosphere during strong geomagnetic storms, J. Geophys. Res., 110, A03208, https: / / doi . org/10.1029/2004 JA010798 